\title{
Reciprocity Calibration of Hydrophones in High Intensity Focused Ultrasound Field
}

\author{
Longyang Jia ${ }^{1,2}$, Wende Shou ${ }^{2}$, Bing $\mathrm{Hu}^{1,2, *}$ \\ ${ }^{1}$ Department of Ultrasound in Medicine, Shanghai Jiao Tong University Affiliated Sixth People's Hospital, Shanghai, China \\ ${ }^{2}$ Shanghai Institute of Ultrasound in Medicine, Shanghai, China
}

Email address:

jialongyang01@163.com (Longyang Jia),wdshou@163.com (Wende Shou), binghu_stephen@163.com (Bing Hu)

${ }^{*}$ Corresponding author

\section{To cite this article:}

Longyang Jia, Wende Shou, Bing Hu. Reciprocity Calibration of Hydrophones in High Intensity Focused Ultrasound Field. American Journal of Physics and Applications. Vol. 8, No. 2, 2020, pp. 19-24. doi: 10.11648/j.ajpa.20200802.11

Received: March 26, 2020; Accepted: April 22, 2020; Published: May 11, 2020

\begin{abstract}
The primary problem of safety and efficiency for the high intensity therapeutic ultrasound (HITU) is the acoustic measure and dose control. The key technique is the pressure and intensity in the acoustic field especial in the focal region using the small calibrated hydrophone. The calibration accuracy of the used hydrophone is very important for HITU. Although the small hydrophone calibration has realized but there was no report of the hydrophone calibration in high pressure field. In this paper, our objective is to develop an absolute calibration method for the measurement of free field voltage sensitivity of hydrophone for high intensity focused ultrasound. First the acoustic pressure at the focal point by the self-reciprocity method of spherically curved auxiliary transducer is calibrated, then the free field voltage sensitivity of hydrophone at the geometric focal point of the calibrated pressure is obtained. The spatial average effect of acoustic pressure on hydrophone surface at the focal point is theoretically modified, and the expression and value table of correction coefficient of spatial average effect of hydrophone are given. The maximum acoustic pressure measured at the focal point was up to $5.58 \mathrm{MPa}\left(1.02 \mathrm{~kW} / \mathrm{cm}^{2}\right)$ and used to calibrate a hydrophone from $0.95 \mathrm{MHz}$ to $1.10 \mathrm{MHz}$ with maximum local distortion parameter 0.72 . The results show the rationality and feasibility of the measurement principle and method.
\end{abstract}

keywords: High Intensity Focused Ultrasound (HIFU), Self-reciprocity Method, Hydrophone Calibration

\section{Introduction}

High intensity focused ultrasound (HIFU) has been widely used in clinical treatment. The acoustic filed performance is a decisive factor that governs the device's range of applicability, efficiency and quality control in manufacturing and application of HIFU therapeutic instrument. With the improvement of sound field theory and the progress of micro hydrophone technology, the measurement technology of spherical focused ultrasonic field has been mature, and the national and international standard have been established. [1, 2] In 2002, W Shou proposed the absolute calibration method of transmitting voltage (current) response and receiving voltage sensitivity of spherical focused transducer, and then it has been applied to acoustic power measurement and hydrophone calibration.[3-6] GB/T19890-2005 [7] is the first national standard for HIFU measurement in the world, it was cited by the IEC and NPL
(British National Physical Laboratory) technical reports [8-9], which stipulates that hydrophone method is the standard method of HIFU acoustic field parameter measurement. Sensitivity is an important performance parameter of hydrophone, and it is the basis of hydrophone used for acoustic field measurement. Therefore, in order to ensure the accuracy of HIFU acoustic field measurement, it is necessary to calibrate the sensitivity of high-intensity hydrophone.

There are no standardized measurement methods applied for calibration of hydrophone under high acoustic pressure. In this paper, a feasible absolute calibration method for measuring the free field voltage sensitivity of hydrophones in high intensity focused ultrasound field was firstly proposed, using self-reciprocity method of spherically curved transducer to calibrate the transmitting response to current (voltage) and the pressure at the geometric focal point of an auxiliary transducer under high power excitation, then calibrating the free field voltage sensitivity of the hydrophone at the geometric focal point. 


\section{Calibrate the High Pressure of the Auxiliary Transducer of High Intensity Focused Ultrasound}

HIFU concentrates ultrasonic waves into the body through certain means (acoustic lens, concave spherically self-focusing, electron focusing, etc.) to form a high-intensity focused region in ultrasonic sound field. The mechanical effect, thermal effect and cavitation effect of ultrasound can be used to achieve high temperature at the focal point instantly $(0.5 \sim 2 \mathrm{~s})$, so as to achieve the purpose of inactivating tumor tissue and avoid damage to surrounding normal cells. Currently, the acoustic power of high intensity focused ultrasound used clinically is usually within the range of hundreds of $\mathrm{W} / \mathrm{cm}^{2}$, therefore, we need high-volume powered hydrophone which have good linearity for high acoustic pressure field, temperature stability, a definite frequency response range, high temperature and impact resistances. At present, there is no perfect special needle hydrophone for high intensity focused ultrasound field measurement except expensive fiber optic sensor. The existing commercial PVDF hydrophone can't be used for high intensity acoustic field measurement for thermal damage. The dynamic range of piezoelectric ceramic hydrophone is infinite and its active element is easily damaged in high pressure. The hydrophone used in this experiment is a new specially designed PVDF high-intensity needle hydrophone of diameter $0.6 \mathrm{~mm}$ in applying for a patent. The PVDF hydrophone is of the tolerability of the short time high temperature and high pressure of HIFU but with low sensitivity $[10,11]$. L Li conducted the plane scanning method in the focal plane of HIFU field to calibrate the HIFU hydrophone where the source acoustic power was measured by radiation force balance [12-15]. But it is very time-consuming and with more error. Although the hudrophone was used to measure the pressure at focal point for 7 HIFU tumor therapeutic equipments (the maximum output power up to $400 \mathrm{~W}$ ) in 2004-2005 and the National Standard of HIFU measurement was drafted in China in 2005, the real maximum pressure can't be still gotten simply so there was no feasible primary method of the hydrophone calibration in high pressure field. The key technique is to calibrate the high pressure of the auxiliary transducer of HIFU. Applying IEC TS 62903-2018 the high-pressure source has been calibrated as expressed below.

\subsection{Measurement of Transmitting Response to Voltage of High Intensity Focused Transducer}

The working frequency of high-intensity focused ultrasound is usually about $1 \mathrm{MHz}$, therefore, the PA885 spherically curved focused transducer of PA company was selected in the experiment, with a resonant frequency of $1 \mathrm{MHz}$, a nominal aperture of $60 \mathrm{~mm}$ and a geometric focal length of $75 \mathrm{~mm}$. The degassed water of oxygen concentration 2.61 ppm is used-to avoid cavitation during calibration。

Measure the effective half-aperture of transducer according to IEC62903. The measurement apparatuses are arranged as shown in Figure 1. The hydrophone measures scanning of hydrophone along the $\mathrm{x}$ axis and the $\mathrm{y}$ axis in the focal plane $(x$, $y, F_{\text {geo }}$ ) determined two pairs of $-3 \mathrm{~dB}$ and $-6 \mathrm{~dB}$ beam width respectively, and their average value are $W_{\mathrm{pb} 3}=2.17 \mathrm{~mm}$, $W_{\mathrm{pb6}}=2.92 \mathrm{~mm}$, the effective radius of the transducer was calculated by $a=\left(0.5 F_{\text {geo }} \lambda / \pi\right)\left[\left(1.62 / w_{\mathrm{pb} 3}\right)+(2.22 /\right.$ $\left.\left.w_{\mathrm{pb} 6}\right)\right]$ equal to $26.67 \mathrm{~mm}$. Calculated by $\beta=\arcsin (a /$ $\left.F_{\text {geo }}\right)$, the focus half-angle is $20.9^{\circ}$, and the effective area obtained from $A=2 \pi F_{\text {geo }}{ }^{2}(1-\cos \beta)$ is $2.35 \times 10^{-3} \mathrm{~m}^{2}$ where $F_{\text {geo }}$ is the geometric focal length, i.e. radius of curvature of the radiation surface $R, \lambda$ is the wavelength.

The transducer and the reflector are arranged in the water tank as shown in Figure 2. The power amplifier used is ELECTRONICS \& INNOVATION MODEL: 1020L. The current monitor is Current Monitor 411 (Beatson Electronics, INC U.S.A.). The oscilloscope is Agilent DSO-X2002A. Adjust the transducer's position making the distance to reflector equal to $F_{\text {geo }}$ and azimuth, elevation to maximize the first echo amplitude received. The three-way switch was placed on 8 to measure the exciting voltage amplitude $U_{\mathrm{T}}$, the first echo voltage amplitude $U_{1}$, exciting current amplitude $I_{T}$ and the first echo current amplitude $I_{\text {echo }}$. The exciting voltage and working frequency of the transducer remained constant, then put this switch on $d$ to measure the open-circuit voltage amplitude of the power amplifier $U_{0}$.

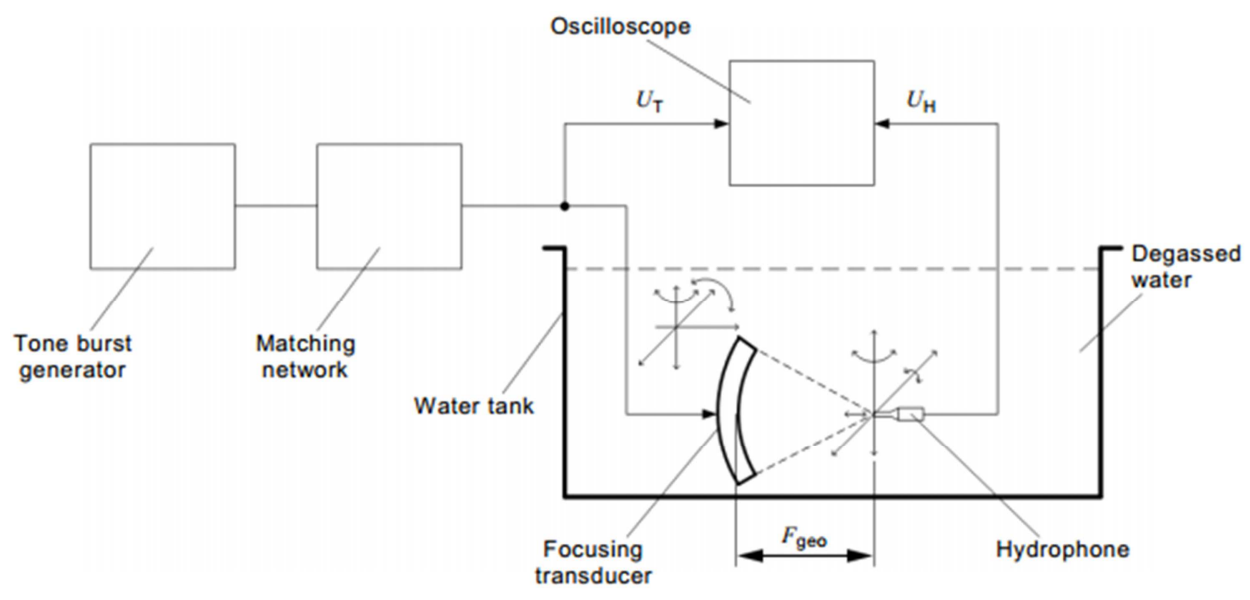

Figure 1. Scheme of the measurement apparatus for determining the effective half-aperture of a transducer [5]. 


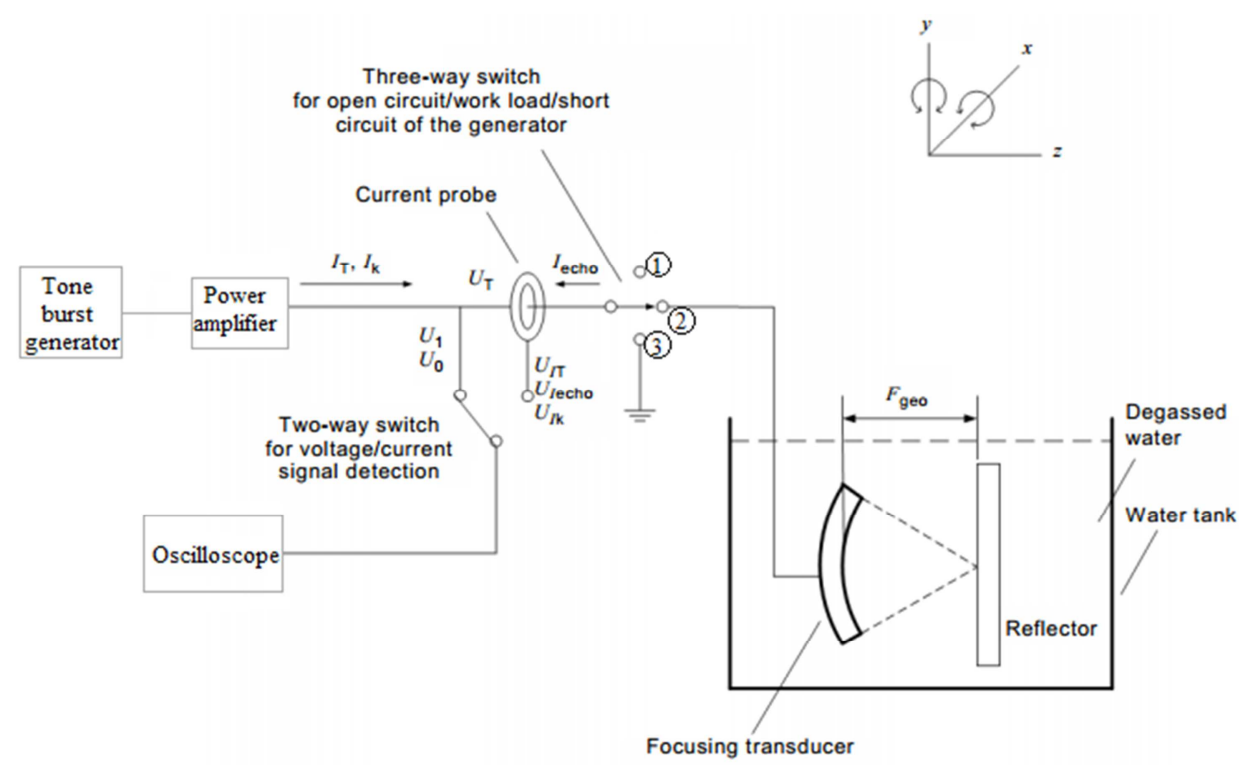

Figure 2. Scheme of free-field self-reciprocity system applied to a spherically curved transducer [2].

The maximum transmitting response at geometric focus to voltage is given by [5]

$$
\begin{gathered}
S_{v f}=\frac{p_{f}}{U_{T}}=k h \sqrt{\frac{\rho c U_{0 r m s} I_{\text {echorms }}}{A r_{a v} G_{s f} U_{T}^{2}}} \\
p_{f}=k h \sqrt{\frac{\rho c U_{0 r m s} I_{\text {echorms }}}{A r_{a v} G_{s f}}} e^{\alpha R}
\end{gathered}
$$

where, $p_{f}$ is the acoustic pressure at the geometric focus, $k=2 \pi / \lambda$ is circular wave number, $h=R(1-\cos \beta)$ is the height at the center of the spherical segment, $r_{a v}(\beta)$ is the average amplitude reflection coefficient on the reflector for the spherically curved transducer and its value is $0.928, G_{s f}$ is the diffraction correction coefficient of the spherically curved transducer and $G_{s f}=0.886, \rho, c$ is the mass density and acoustic velocity of water respectively. $\alpha$ is the acoustic attenuation coefficient in water, the acoustic attenuation $e^{\alpha R}$ for $1 \mathrm{MHz}$ ultrasound and distance $R=75 \mathrm{~mm}$ in water can be ignored.

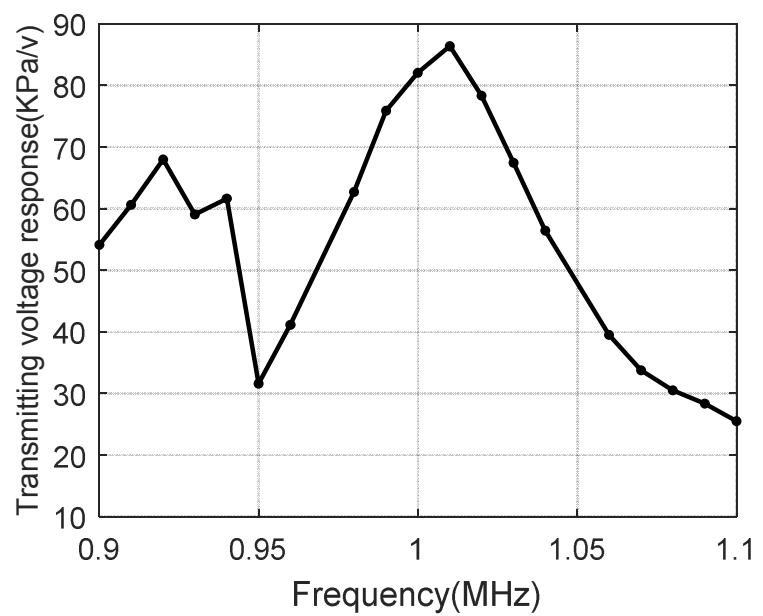

Figure 3. The frequency response curve of the transmitting voltage response $S_{v f}$ at the geometric focus of the transducer PA885.
The curves of the transmitting voltage response $S_{v f}$ at the geometric focus of the transducer vs frequency is shown in Figure 3, the maximum value of $S_{v f}$ at $1.01 \mathrm{MHz}$ is 85.8 $\mathrm{kPa} / \mathrm{V}$.

\subsection{Nonlinear Distortion of Ultrasound at the Transducer Focus}

Calculating the local distortion parameter $\sigma_{q}$ to determine nonlinear distortion of ultrasound at the transducer focus [16].

$$
\sigma_{q}=z p_{m} \frac{2 \pi f_{a w f} \beta}{\rho c^{3}} \frac{1}{\sqrt{F_{a}}}
$$

where $z$ is the axial distance of the point of interest to the transducer face, i.e. the geometric focal length $F_{\text {geo }} ; p_{\mathrm{m}}$ is the mean-peak acoustic pressure at the point in the acoustic field corresponding to the spatial-peak temporal-peak acoustic pressure; $\beta$ is the nonlinearity parameter $(\beta=1+B / A=3.5$ for pure water at $\left.20^{\circ} \mathrm{C}\right), f_{\text {awf }}$ is the acoustic-working frequency; $F_{\text {a }}$ is the local area factor, $F_{a}=\sqrt{0.69 A_{\mathrm{SAeff}} / A_{\mathrm{b},-6 \mathrm{~dB}}}, A_{\mathrm{SAeff}}$ is the source aperture area, i.e. the effective area of the transducer; $A_{b,-6 d B}$ is beam area at the $-6 \mathrm{~dB}$ level. Figure 4 shows the relationship between the focal acoustic pressure $p_{\mathrm{f}}$ and the effective exciting voltage $U_{\text {Trms }}$ of the transducer at $1 \mathrm{MHz}$. In this figure, when the effective exciting voltage $U_{\text {Trms }}=42.29 \mathrm{~V}$, the focal acoustic pressure $p_{\mathrm{f}}$ is $3.58 \mathrm{MPa}$, $\sigma_{q}=0.46(<0.5)$, and the focal acoustic pressure caused by nonlinear distortion effect differs by less than $5 \%$ from the value in the absence of nonlinear effect. If $0.5<\sigma_{q}<1.5$, this difference is between $5 \%$ and $25 \%$. When $U_{\text {Trms }}=$ $65.25 \mathrm{~V}, p_{\mathrm{f}}=5.58 \mathrm{MPa}$ and $\sigma_{\mathrm{q}}=0.72$ the acoustic pressure waveform at focal point appears obvious distortion with the estimated pressure deviation about $10 \%$ caused by nonlinearity, but the relation of focal pressure and exciting voltage remains good linearity as shown in Figure 4 and that of acoustic power and exciting voltage squared is also linear, see Figure 5. 


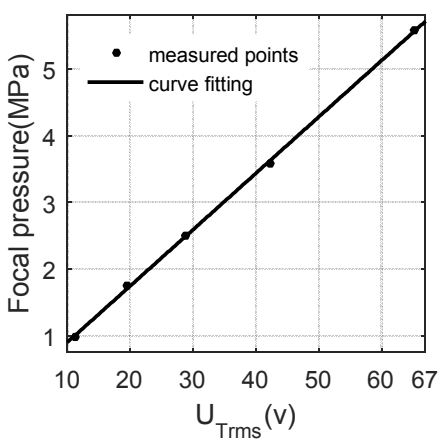

Figure 4. The fitting line of the focal acoustic pressure $p_{f} v s$. the effective exciting voltage $U_{\text {Trms }}$ of the transducer at $1 \mathrm{MHz}$ frequency.

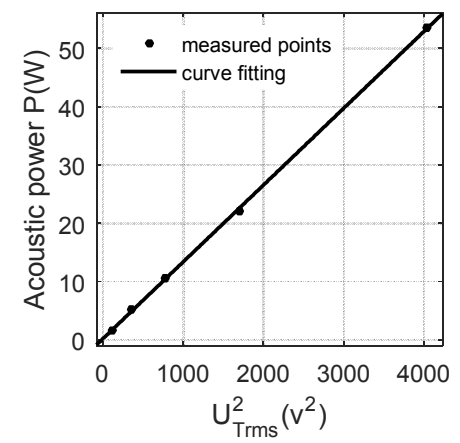

Figure 5. The fitting line of output power $P$ vs. exciting voltage squared $U_{\text {Trms }}^{2}$.

\section{Hydrophone Calibration}

\subsection{The Correction Coefficient for the Spatial Average Effect of the Acoustic Pressure over the Hydrophone Surface if the Hydrophone were Removed}

The acoustic pressure received by the hydrophone is not the value at the geometric focus in free field, but the average acoustic pressure over the hydrophone surface if it were removed, which affects the sensitivity of the hydrophone to a certain extent, so it needs to be corrected. The correction factor of the pressure average effect of hydrophone $G_{2}$ can be derived:

$$
G_{2}=\frac{\overline{p_{H}}}{p_{f}}=2 \cot ^{2} \psi_{m} \int_{0}^{\psi_{m}} \operatorname{jinc}(k a \sin \psi) \tan \psi \sec ^{2} \psi d \psi
$$

where

$$
\begin{gathered}
\overline{p_{H}}=\frac{p_{f}}{A_{m}} \int_{0}^{\psi_{m}} j \operatorname{jinc}(k a \sin \psi) \mathrm{dA} \\
d A=2 \pi R^{2} \tan \psi d(\tan \psi) \\
A_{m}=\pi R^{2} \tan ^{2} \psi_{m}
\end{gathered}
$$

$\overline{p_{H}}$ is the average free-field pressure over the effective area of the active element of the hydrophone in the middle of the geometric focal plane if the hydrophone were removed. $\psi_{\mathrm{m}}=\arcsin \left(a_{H} / R\right)$ is the half-aperture angle of the hydrophone for the center of the focusing transducer. $a_{H}$ is the effective radius of the active element of the hydrophone.

Obviously, $G_{2}$ is the function of parameters $k a$ and $\psi_{\mathrm{m}}$, the dependence of $G_{2}$ on $\psi_{\mathrm{m}}$ at several values of $k a$ was shown in Figure 6. The values of function $G_{2}\left(k a, \psi_{\mathrm{m}}\right)$ were list in Table 1, when the parameters $k a$ and $\psi_{\mathrm{m}}$ in the table are close to the corresponding values of the measured transducers, $G_{2}$ can be obtained by linear interpolation using the data in the Table 1 .

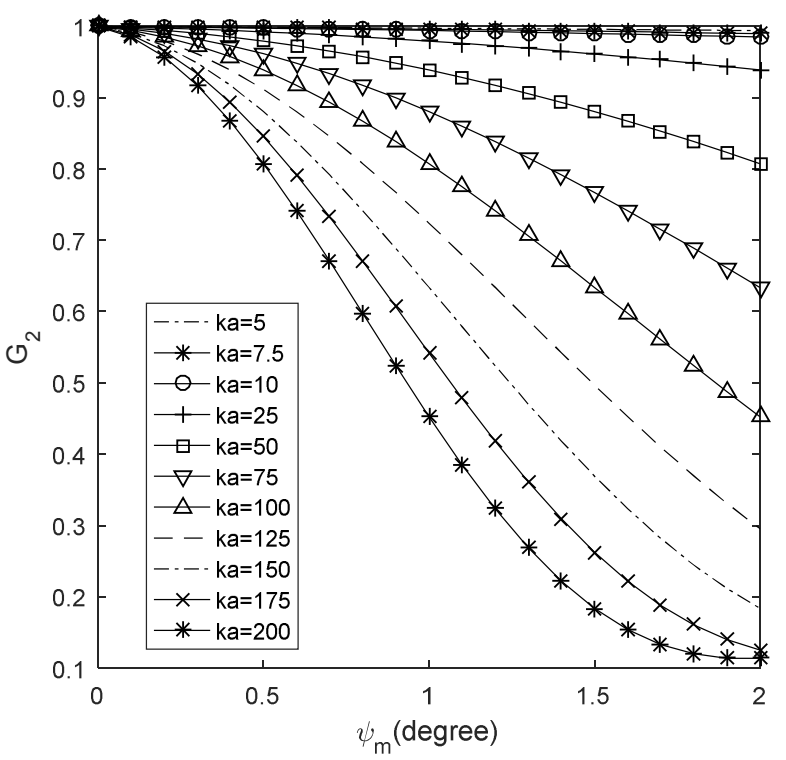

\begin{tabular}{|c|c|c|c|c|c|c|c|c|c|c|c|c|c|c|c|c|c|c|c|c|}
\hline $\begin{array}{l}\psi_{m}\left(^{\circ}\right) \\
\boldsymbol{k}^{a}\end{array}$ & 0 0.1 & 0.2 & 0.3 & 0.4 & 0.5 & 0.6 & 0.7 & 0.8 & 0.9 & 1 & 1.1 & 1.2 & 1.3 & 1.4 & 1.5 & 1.6 & 1.7 & 1.8 & 1.9 & 2 \\
\hline 5 & 10.9998 & 0.9996 & 0.9994 & 0.9991 & 0.9989 & 0.9986 & 0.9984 & 0.9981 & 0.9978 & 0.9975 & $0.9972 \mathrm{c}$ & 0.9969 & 0.9966 & 0.9963 & 0.9959 & 0.9956 & 0.9952 & 0.9948 & 30.9945 & 0.9941 \\
\hline 7.5 & $\begin{array}{ll}10.9997\end{array}$ & 0.9994 & 0.9990 & 0.9987 & 0.9983 & 0.9979 & 0.9974 & 0.9970 & 0.9965 & 0.9960 & 0.9955 & 0.9950 & 0.9944 & 0.9938 & 0.9932 & 0.9926 & 0.992 & 0.9913 & 0.9907 & 0.9900 \\
\hline 10 & 10.9996 & 0.9991 & 0.9987 & 0.9981 & 0.9976 & 0.9970 & 0.9964 & 0.9957 & 0.9950 & 0.9943 & 0.9935 & 0.9927 & 0.9919 & 0.9910 & 0.9901 & 0.9892 & 0.9882 & 0.9872 & 0.9861 & 0.9850 \\
\hline 25 & 10.9989 & 0.9976 & 0.9961 & 0.9943 & 0.9924 & 0.9902 & 0.9879 & 0.9853 & 0.9825 & 0.9795 & $0.9763 c$ & 0.9729 & 0.9693 & 0.9655 & 0.9615 & 0.9574 & 0.953 & 0.9484 & 0.9437 & 0.9388 \\
\hline 50 & 10.9976 & 0.9944 & 0.9903 & 0.9853 & 0.9796 & 0.9730 & 0.9656 & 0.9575 & 0.9486 & 0.9389 & 0.9286 & 0.9175 & 0.9058 & 0.8935 & 0.8805 & 0.8670 & 0.8529 & 0.8382 & 0.8231 & 0.8075 \\
\hline 75 & 10.9961 & 0.9903 & 0.9826 & 0.9730 & 0.9617 & 0.9486 & 0.9339 & 0.9176 & 0.8998 & 0.8806 & 0.8601 & 0.8383 & 0.8155 & 0.7916 & 0.7669 & 0.741 & 0.7152 & 0.6885 & 0.661 & 0.6341 \\
\hline 100 & 10.9944 & 0.9853 & 0.9730 & 0.9575 & 0.9390 & 0.9176 & 0.8936 & 0.8671 & 0.8384 & 0.8077 & $0.7753 c$ & 0.7414 & 0.7064 & 0.6706 & 0.6341 & 0.5973 & 0.5605 & 0.5240 & 0.4879 & 0.4527 \\
\hline 125 & $\begin{array}{ll}10.9924 \\
\end{array}$ & 0.9796 & 0.9617 & 0.9390 & 0.9119 & 0.8806 & 0.8457 & 0.8077 & 0.7669 & 0.7241 & 0.6796 & 0.6341 & 0.5881 & 0.5422 & 0.4969 & 0.4527 & 0.4100 & 0.3693 & 0.3309 & 0.2952 \\
\hline 150 & 10.9903 & 0.973 & 0.9486 & 0.9176 & 0.8806 & 0.8384 & 0.7917 & 0.741 & 0.6886 & 0.6341 & 0.5789 & 0.5240 & 0.4702 & 0.4184 & 0.3693 & 0.323 & 0.2817 & 0.2443 & 0.2115 & 0.1836 \\
\hline 175 & 10.9879 & 0.9657 & 0.9339 & 0.8936 & 0.8458 & 0.7917 & 0.7328 & 0.6706 & 0.6066 & 0.5422 & 0.4791 & 0.4184 & 0.3614 & 0.3091 & 0.2624 & $0.221 \mathrm{C}$ & 0.1879 & 0.1605 & 0.1398 & 0.1253 \\
\hline 200 & 10.9853 & 0.9575 & 0.9176 & 0.8671 & 0.8077 & 0.7415 & 0.6706 & 0.5974 & 0.5240 & 0.4527 & 0.3853 & 0.3235 & 0.2688 & 0.2219 & 0.1836 & 0.1539 & 0.1328 & 0.1197 & 0.1136 & 0.1136 \\
\hline
\end{tabular}

Figure 6. Relationship of $G_{2}$ and $\psi_{m}$ at several values of $k a$.

Table 1. The values of correction coefficient $G_{2}\left(k a, \psi_{m}\right)$ for the spatial average effect of the free-field acoustic pressure over the hydrophone surface if it were removed. 


\subsection{Hydrophone Calibration Experiment}

Using the calibrated focusing transducer as an acoustic source, remove the flat reflector from the tank and place the hydrophone to be calibrated near the geometric focus, repeatedly adjust the position and direction of the hydrophone so that its maximum sensitivity direction is collinear with the beam axis of the focus transducer, and the distance between them remains $F_{\text {geo }}$, the cable-end loaded voltage amplitude $E_{\mathrm{H}}$ reaches maximum and read by the oscilloscope (Agilent DSO-X2002A, probe's electrical load: $10 \mathrm{M} \Omega, 11 \mathrm{pF}$ ).

The relationship between the root mean square (rms) excitation voltage $U_{\text {Trms }}$ and the corresponding hydrophone rms cable-end output loaded voltage $E_{\text {Hrms }}$ is shown in Figure 7 , and the linearity is $5.36 \%$ by least squares fitting.

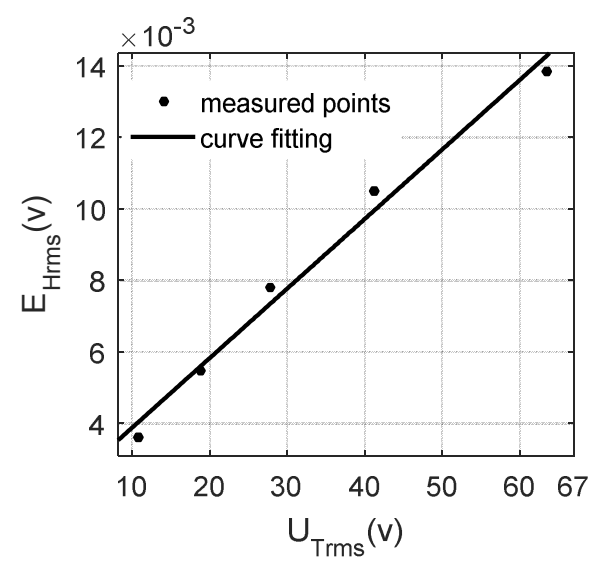

Figure 7. The rms hydrophone cable-end loaded output voltage $E_{H r m s}$ vs. the exciting voltage $U_{\text {Trms }}$.

The focal intensity is $I_{\mathrm{f}}=q p_{\mathrm{f}}^{2} /(2 \rho c)$, where $q=(1+\cos$ $\beta) / 2$ is the cosine-function value of the phase difference between the particle velocity and the acoustic pressure at the geometric focus, i.e. the acoustic power factor [2]. Figure 8 is the relationship between the focal acoustic intensity and the rms excitation voltage.

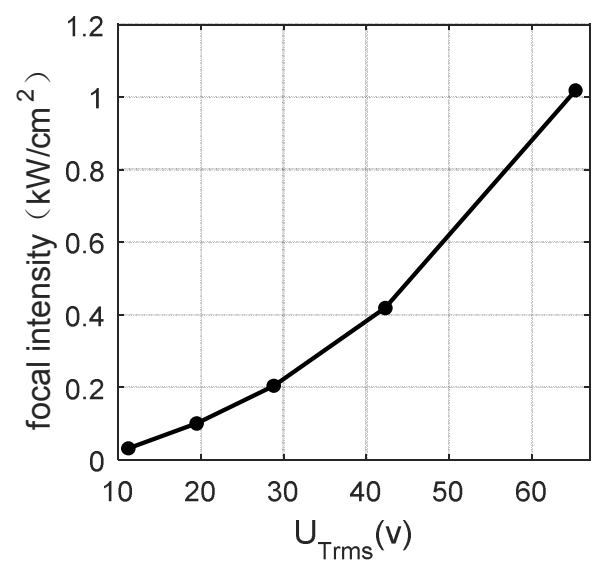

Figure 8. The focal intensity vs. rms exciting voltage $U_{\text {Trms }}$.

Figure 8 shows that the focal acoustic intensity increases with the square law when the excitation voltage increases. The focal acoustic intensity can reach $1.02 \mathrm{~W} / \mathrm{cm}^{2}$ at $p_{\mathrm{f}}=5.58 \mathrm{MPa}$.

The hydrophone was placed at the geometric focus, and the cable-end loaded output voltage of the hydrophone was measured. Then the free field cable-end loaded voltage sensitivity was given by

$$
M_{\mathrm{L}}=E_{\mathrm{H}} /\left(p_{\mathrm{f}} \cdot G_{2}\right)
$$

The frequency response of the free field cable-end voltage sensitivity of the hydrophone is shown in Figure 9.

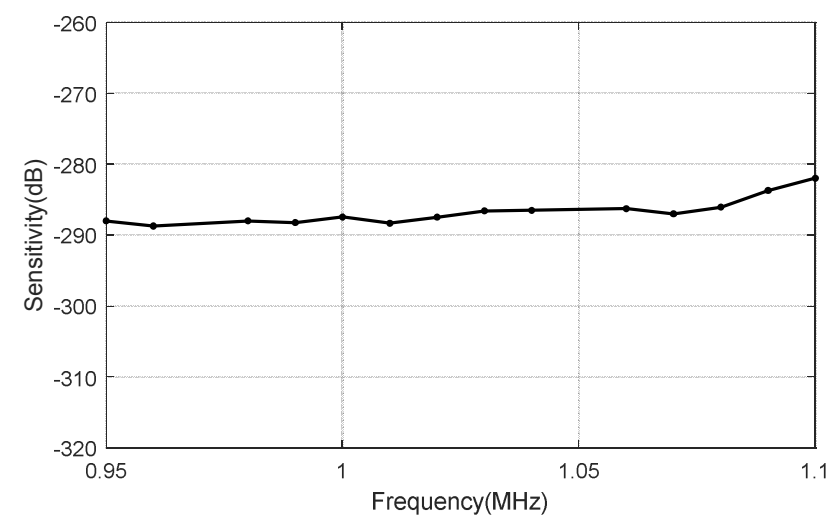

Figure 9. The free field cable-end loaded voltage sensitivity level of hydrophone.

From Figure 9, when the frequency is $1 \mathrm{MHz}$, the free field cable-end loaded voltage sensitivity level of hydrophone is $-287.5 \mathrm{~dB}$ reference: $1 \mathrm{~V} / \mu \mathrm{Pa}$. In the frequency range $0.95 \mathrm{MHz}$ to $1.06 \mathrm{MHz}$ the frequency response of sensitivity is relatively flat within a range of $\pm 1.33 \mathrm{~dB}$.

\section{Conclusion}

A new calibration method for the hydrophone used for HIFU was presented and carried out, which is based on the self-reciprocity method of a high-pressure focusing transducers. The values of correction factor $G_{2}\left(k a, \psi_{\mathrm{m}}\right)$ for spatial average effect of pressure over the hydrophone surface were given. The acoustic pressure at the focal point calibrated by experiments can be up to $5.58 \mathrm{MPa}\left(1.02 \mathrm{~kW} / \mathrm{cm}^{2}\right)$, the calibration of the hydrophone used in HIFU field has been realized. The applications of fully degassed water and the burst source can effectively increase the cavitation threshold so that it did not happened in calibration. At normal temperature, the cavitation threshold of tap water is about $-1 \mathrm{MPa}$, and that of degassed water is about $-30 \mathrm{MPa}$. In the experiment the maximum pressure $5.58 \mathrm{MPa}$ is much less than the threshold but has introduced $10 \%$ nonlinear change of the local field as $\sigma_{\mathrm{m}}=0.72>0.5$. It is obvious that the nonlinear distortion effect is the primary obstacle for increasing the maximum pressure in experiment. To increase the upper limit of local distortion paramete $\sigma_{\mathrm{m}}$ in experiment the acoustic source must be optimized. Reducing the geometric focal length, increasing the aperture and the local area factor of the transducer are the effect methods. 


\section{Acknowledgements}

The authors would like to acknowledge the funding and support of National Key Research and Development Project of China (2017YFC0113800; 2017YFC0113801).

\section{References}

[1] GB/T 32522-2016 Acoustics-Electroacoustic characteristics and measurements of piezoeletric focusing ultrasonic transducers (in Chinese) [S].

[2] IEC TS 62903 Ed. 1.0-2018 Ultrasonics - Measurements of electroacoustical parameters and acoustic output power of spherically curved transducers using the self-reciprocity method [S].

[3] W SHOU, J YAN, H WANG,. Free-field reciprocity calibration in a convergent spherical acoustic wave of a focusing transducer [J]. Chin. Phys. Lett., 19 (8): 1131-1136, 2002.

[4] S Duan, W Shou, and P He et al., " A novel method to measure acoustic power of focusing transducer with spherical surface based on self-reciprocity theorem," Chinese Physics Letters, 22 (1): 146-149, 2005.

[5] Shou W. D., Yu L. L., Duan S. M., et al, Research on electro-acoustic characteristics and measurements of spherically focusing transducer [J] (in Chinese), Technical Acoustics, 000 (005), 379-388, 2013.

[6] G Z Xing, P Yang, W D Shou, M Wang, X J Feng, L B He, Hydrophone reciprocity calibration in spherically focused ultrasonic field from $1 \mathrm{MHz}$ to $15 \mathrm{MHz}[\mathrm{J}]$, Acta Acustica united with Acustica, 105: 273-283, 2019.

[7] GB/T 19890-2005 Acoustics- Measurements of acoustic power and field characteristics of high intensity focused ultrasound (HIFU), (in Chinese) [S].

[8] Shaw A, tea Haar Gail, NPL REPORT DQL AC-015: Requirements for Measurement Standards in High Intensity Focused Ultrasound (HIFU) Fields, 2006. [S].

[9] IEC REPORT 62649-2010: Ultrasonics-Requirements for Measurement Standard of High Intensity Therapeutic Ultrasound (FITU) Equipments. [S].

[10] Granz B. PVDF hydrophone for the measurement of shock waves [J], International Symposium on Electrets. IEEE, 1988.

[11] Robinson S, Preston R. PVDF reference hydrophone development in the UK-from fabrication and lamination to use as secondary standards [J]. IEEE Transactions on Ultrasonics, Ferroelectrics, and Frequency Control, 47 (6): P. 1336-1344, 2000.

[12] L Li, The study of hydrophone for the measurement of High intensity focused ultrasound and calibration techniques, ( Academic dissertation for MA. Eng in SJTU, China) 2003.

[13] Gélat, Pierre, Shaw, A. Relationship between acoustic power and acoustic radiationforce on absorbing and reflecting targets for spherically focusing radiators [J]. Ultrasound Med Biol, 41 (3): 832-844, 2015.

[14] Shou W, Huang X, Duan S, et al., Acoustic power measurement of high intensity focused ultrasound in medicine based on radiation force [J]. Ultrasonics, 44 (8): e17-e20, 2006.

[15] Shou W, Recall HIFU measurement and talk about standard innovation [J] (in Chinese), International Medical Devices, 17 (2): 70-74, 2011.

[16] IEC62127-1: 2013 Ultrasonics- Hydrophones- Prat1: Measurement and characteristics of medical ultrasonic fields up to $40 \mathrm{MHz}$. [S]. 